\title{
CONFORMALLY FLAT MANIFOLDS AND A PINCHING PROBLEM ON THE RICCI TENSOR
}

\author{
SAMUEL I. GOLDBERG ${ }^{1}$ AND MASAFUMI OKUMURA
}

\begin{abstract}
There is a formal similarity between the theory of hypersurfaces and conformally flat $d$-dimensional spaces of constant scalar curvature provided $d \geqslant 3$. For, then, the symmetric linear transformation field $Q$ defined by the Ricci tensor satisfies Codazzi's equation $\left(\nabla_{X} Q\right) Y=\left(\nabla_{Y} Q\right) X$. This observation leads to a pinching theorem on the length of the Ricci tensor.
\end{abstract}

1. Statement of results. Recently, one of the authors [1] obtained

THEOREM G. Let $M$ be a d-dimensional compact conformally flat manifold with definite Ricci curvature. If the scalar curvature $r$ is constant and if the square of the length of the Ricci tensor is not greater than $r^{2} /(d-1), d \geqslant 3$, then $M$ is a space of constant curvature.

Note that the square length of the Ricci tensor is greater than or equal to $r^{2} / d$, so the Ricci tensor has been "pinched". In the present paper the following two theorems are proved, the first of which generalizes Theorem G.

THEOREM 1. Let $M$ be a d-dimensional compact conformally flat manifold with constant scalar curvature $r$. If the length of the Ricci tensor is less than $r / \sqrt{d-1}, d \geqslant 3$, then $M$ is a space of constant curvature.

THEOREM 2. In a d-dimensional compact conformally flat manifold $M$, if the length of the Ricci tensor is constant and less than $r / \sqrt{d-1}$, then $M$ is a space of constant curvature.

2. Conformally flat manifolds. Let $M$ be a Riemannian manifold of dimension $d \geqslant 3$. We cover $M$ by a system of local coordinate neighborhoods $\left\{U, x^{h}\right\}$, and denote by $g_{j i}, \nabla_{j}, R_{k j i}{ }^{h}$, and $R_{j i}$ the Riemannian metric, the operator of covariant differentiation in terms of the Riemannian connection, the curvature tensor and the Ricci tensor, respectively. We say that $M$ is conformally flat if its Riemannian metric is conformally related to a locally Euclidean metric. In a conformally flat manifold,

Received by the editors June 9, 1975 and, in revised form, October 15, 1975.

AMS (MOS) subject classifications (1970). Primary 53A30, 53B20, 53C20.

Key words and phrases. Conformally flat manifolds, Ricci tensor, scalar curvature.

${ }^{1}$ Research partially supported by the National Science Foundation.

(c) American Mathematical Society 1976 


$$
\begin{aligned}
R_{k j i h}= & \frac{1}{d-2}\left(R_{j i} g_{k h}-R_{k i} g_{j h}+g_{j i} R_{k h}-g_{k i} R_{j h}\right) \\
& -\frac{r}{(d-1)(d-2)}\left(g_{j i} g_{k h}-g_{k i} g_{j h}\right),
\end{aligned}
$$

$$
\nabla_{k} R_{j i}-\nabla_{j} R_{k i}=\frac{1}{2(d-1)}\left(g_{j i} \nabla_{k} r-g_{k i} \nabla_{j} r\right) .
$$

As a consequence of (2.1) and (2.2), in a conformally flat manifold, the Laplacian of the square length of the Ricci tensor becomes

$$
\begin{aligned}
\frac{1}{2} \Delta \operatorname{tr} Q^{2}= & \frac{1}{2(d-1)}\left\{r g^{j i}+(d-2) R^{j i}\right\} \nabla_{j} \nabla_{i} r \\
& +P /(d-2)+g(\nabla Q, \nabla Q),
\end{aligned}
$$

where

$$
P=d \operatorname{tr} Q^{3}-\frac{2 d-1}{d-1} r \operatorname{tr} Q^{2}+\frac{r^{3}}{d-1} .
$$

As in [1], $P$ is nonnegative if $\sqrt{ } \operatorname{tr} Q^{2}<r / \sqrt{d-1}$. Thus

$$
\frac{1}{2} \Delta \operatorname{tr} Q^{2} \geqslant \frac{1}{2(d-1)}\left\{r g^{j i}+(d-2) R^{j i}\right\} \nabla_{j} \nabla_{i} r+g(\nabla Q, \nabla Q) .
$$

3. Proofs of theorems. The following lemma is fundamental and may be found in [2].

LEMMA. Let $a_{1}, \ldots, a_{d}$ and $k$ be real numbers satisfying the inequality

$$
\sum_{i=1}^{d} a_{i}^{2}+k<\frac{1}{d-1}\left(\sum_{i=1}^{d} a_{i}\right)^{2} .
$$

Then, for any pair of distinct $i$ and $j=1, \ldots, d, k<2 a_{i} a_{j}$.

If $M$ satisfies the conditions of Theorem 1, the lemma shows that the eigenvalues of the Ricci tensor all have the same sign. Thus the Ricci tensor is positive definite. Consequently, by Theorem G, $M$ is a space of constant curvature. If $M$ satisfies the conditions of Theorem 2, then by the lemma, the Ricci tensor is positive definite, and so is $r g^{j i}+(d-2) R^{j i}$. Thus, (2.4) yields the inequality

$$
\left\{r g^{j i}+(d-2) R^{j i}\right\} \nabla_{j} \nabla_{i} r \leqslant 0 .
$$

Since $M$ is compact, E. Hopf's well-known theorem says $r$ is constant. Hence, $M$ satisfies the conditions of Theorem 1 .

The inequality in Theorems 1 and 2 is the best possible. For, if $M$ $=S^{d-1} \times S^{1}$, where $S^{d-1}$ is the constant curvature sphere, then $M$ is conformally flat, $r$ is constant and $\operatorname{tr} Q^{2}=r^{2} /(d-1)$. 


\section{BIBLIOGRAPHY}

1. S. I. Goldberg, On conformally flat spaces with definite Ricci curvature. II, Ködai Math. Sem. Rep. (to appear).

2. M. Okumura, Submanifolds and a pinching problem on the second fundamental tensors, Trans. Amer. Math. Soc. 178 (1973), 285-291. MR 47 \# 5793.

Department of Mathematics, University of Illinois, Urbana, Illinois 61801

Department of Mathematics, Sattama University, Saitama, Japan 